\title{
Wave Operators and the Incompressible Limit of the Compressible Euler Equation
}

\author{
Hiroshi Isozaki \\ Department of Mathematics, Osaka University, Toyonaka, 560 Japan
}

\begin{abstract}
In an exterior domain in $R^{n}(n \geqq 2)$, the solution of the compressible Euler equation is shown to converge to that of the incompressible Euler equation when the Mach number tends to 0 . The initial layer appears.
\end{abstract}

\section{Introduction}

In our previous work [8], we have shown that the solution of the compressible Euler equation in an exterior domain in $R^{3}$ converges to that of the incompressible Euler equation when the Mach number tends to 0 even if the initial velocity is not divergence free. The aim of this article is to generalize this result for $R^{n}(n \geqq 2)$ and also to provide a simpler proof.

We consider the movement of an ideal fluid in a domain $\Omega$ in $R^{n}(n \geqq 2)$ exterior to a bounded obstacle. Let $P$ be its pressure and $V$ the velocity. Then the Euler equation is written as

$$
\begin{aligned}
& \partial_{t} P+(V \cdot \nabla) P+\gamma P \nabla \cdot V=0, \\
& \partial_{t} V+(V \cdot \nabla) V+\lambda^{2} P^{-1 / \gamma} \nabla P=0, \\
& v \cdot V=0 \quad \text { on } \quad S,
\end{aligned}
$$

where $\partial_{t}=\partial / \partial t, \gamma$ is a constant $>1, S$ is the boundary of $\Omega$ and $v$ is the outer unit normal to $S$. $\lambda$ is a large parameter proportional to the inverse of the Mach number (see, e.g., [14, p. 52]). We assume that $S$ is smooth and $\Omega$ is arcwise connected, but nothing is assumed on the shape of the boundary. It is convenient to transform the dependent variable $P$ into $Q=\frac{\gamma}{\gamma-1} P^{1-1 / \gamma}$. Then the above equation can be
rewritten as

$$
\begin{gathered}
\partial_{t} Q+(V \cdot \nabla) Q+(\gamma-1) Q \nabla \cdot V=0, \\
\partial_{t} V+(V \cdot \nabla) V+\lambda^{2} \nabla Q=0 .
\end{gathered}
$$

We set $\gamma=2$ for the sake of simplicity. We want to assume that the initial pressure has an asymptotic expansion of the form: Const. $+O\left(\lambda^{-1}\right)$. Therefore, without loss 
of generality, we put $Q=1+p / \lambda$ and correspondingly $V=v$. Then we arrive at the following equation:

$$
\begin{aligned}
& \partial_{t} p+(v \cdot \nabla) p+p \nabla \cdot v+\lambda \nabla \cdot v=0 \\
& \partial_{t} v+(v \cdot \nabla) v \quad+\lambda \nabla p=0 \\
& v \cdot v=0 \quad \text { on } \quad S .
\end{aligned}
$$

Let $H^{m}(\Omega)$ be the usual Sobolev space of order $m$, whose norm is denoted by $\|\cdot\|_{m}$. The following assumptions are imposed on the initial data $p_{0}^{\lambda}$ and $v_{0}^{\lambda}$ :

(A-1) $\left\{\left(p_{0}^{\lambda}, v_{0}^{\lambda}\right) ; \lambda>0\right\}$ is a bounded set in $H^{N+1}(\Omega) \cap L^{1}(\Omega)$, where $N$ is an integer $\geqq[n / 2]+3$.

(A-2) $\left(p_{0}^{\lambda}, v_{0}^{\lambda}\right)$ satisfies the compatibility condition up to order $N+1$.

(A-3) $P_{S} v_{0}^{\lambda} \rightarrow v_{0}^{\infty}$ in $H^{N}(\Omega)$ as $\lambda \rightarrow \infty$, where $P_{S}$ is the projection onto the solenoidal fields to be defined in Sect. 2.

Then our results are:

Theorem A (Uniform Estimates). There exist constants $T>0$ and $\Lambda>0$ such that for any $\lambda>\Lambda$, there exists a unique solution

$$
p^{\lambda}(t), v^{\lambda}(t) \in \bigcap_{k=0}^{N} C^{k}\left(I ; H^{N-k}(\Omega)\right), \quad I=[0, T] .
$$

Moreover, it obeys the following uniform estimate:

$$
\sup _{\lambda>\Lambda, t \in I}\left(\left\|p^{\lambda}(t)\right\|_{N}+\left\|v^{\lambda}(t)\right\|_{N}\right)<\infty
$$

Theorem B (Incompressible Limit). For $0<t \leqq T, p^{\lambda}(t) \rightarrow 0$ and $v^{\lambda}(t) \rightarrow v^{\infty}(t)$ in $H_{\text {loc }}^{N-1}(\bar{\Omega})$ as $\lambda \rightarrow \infty$. Furthermore, $v^{\infty}(t)$ satisfies the incompressible Euler equation

$$
\begin{gathered}
\partial_{t} v^{\infty}+P_{S}\left(v^{\infty} \cdot \nabla\right) v^{\infty}=0 \quad \text { in } \Omega, \quad t \in I, \\
v^{\infty}(0)=P_{S} v_{0}^{\infty}=v_{0}^{\infty} .
\end{gathered}
$$

In [8], we have already proved the similar results in $R^{3}$. The essential point for proving Theorem $\mathrm{B}$ was the decay lemma for the linearized operator of acoustics [8, Lemma 2.1]. However, aside from the interest of its own, the proof is complicated and can not be applied to the 2-dimensional case. In this paper, we shall avoid this difficulty by utilizing the completeness of wave operators for the linearized operator of acoustics in an exterior domain.

The problem of the singular perturbation for non-linear equations of fluids is studied by many authors and is still developing (see the references cited at the end of this paper). One can see an abundance of problems in Majda's book [14, Chap. 2].

\section{Proof of Theorem A}

The proof of Theorem A is almost the same as in [8]. The only difference is the Helmholtz decomposition. Let

$$
C_{0, \sigma}^{\infty}(\Omega)=\left\{w \in C_{0}^{\infty}(\Omega) ; \operatorname{div} w=0\right\},
$$

and $S(\Omega)$ be its closure in $L^{2}(\Omega)^{n}$. Let $G(\Omega)$ be the orthogonal complement of $S(\Omega)$ in $L^{2}(\Omega)^{n}$. Let $P_{G}$ and $P_{S}$ be the projections onto $G(\Omega)$ and $S(\Omega)$, respectively. 
Lemma 2.1. (1) If $w \in S(\Omega)$, div $w=0$ in the distribution sense and $v \cdot w=0$ in $H^{-1 / 2}(S)$.

(2) If $v \in G(\Omega)$, there exists a $\varphi \in H_{\mathrm{loc}}^{1}(\bar{\Omega})$ such that $v=\nabla \varphi$.

For the proof, see [18, p. 9] and [11, p. 27].

Once the above lemma is noticed, the methods in [8, Sects. 1 and 5] need no essential change. Lemmas 1.5 and 1.15 are not necessary in the present case. Lemma 1.7 follows from the estimate of [5], Lemma 5. The Sobolev inequality in (5.1) should be replaced by

$$
\|f g\|_{r} \leqq C\|f\|_{s}\|g\|_{t}, \quad 0 \leqq r \leqq \min \{s, t, s+t-[n / 2]-1\} .
$$

These modifications are sufficient to prove Theorem A, hence we omit the details.

\section{Proof of Theorem B}

For the solution $p^{\lambda}(t), v^{\lambda}(t)$ of (1.1), we set $f^{\lambda}(t)={ }^{t}\left(p^{\lambda}(t), v^{\lambda}(t)\right)$ and

$$
A\left(f^{\lambda}\right)=\left(\begin{array}{cc}
v^{\lambda} \cdot \nabla & p^{\lambda} \nabla \\
0 & v^{\lambda} \cdot \nabla
\end{array}\right), \quad \nabla=\left(\partial / \partial x_{1}, \ldots, \partial / \partial x_{n}\right) .
$$

Let $L$ be the linearized operator of acoustics in $L^{2}(\Omega)$ :

$$
L=-i\left(\begin{array}{cc}
0 & \nabla \\
{ }^{t} \nabla & 0
\end{array}\right)
$$

with the boundary condition $v \cdot v=0$ on $S$. Then one can rewrite (1.1) as

$$
\partial_{t} f^{\lambda}+A\left(f^{\lambda}\right) f^{\lambda}+i \lambda L f^{\lambda}=0
$$

We transform it into the integral equation

$$
\begin{gathered}
f^{\lambda}(t)=e^{-i t \lambda L} f_{0}^{\lambda}-\int_{0}^{t} e^{-i(t-s) \lambda L} A\left(f^{\lambda}(s)\right) f^{\lambda}(s) d s, \\
f_{0}^{\lambda}={ }^{t}\left(p_{0}^{\lambda}, v_{0}^{\lambda}\right) .
\end{gathered}
$$

Let $\Gamma_{0}$ be the projection onto the null space of $L$ and $\Gamma=1-\Gamma_{0}$. They are bounded in $H^{m}(\Omega)(m \geqq 0)$. For $f={ }^{t}(p, v), \Gamma_{0} f={ }^{t}\left(0, P_{S} v\right)$. The first step of the proof is:

Step 1. For $t>0, \Gamma f^{\lambda}(t) \rightarrow 0$ weakly in $L^{2}(\Omega)$ as $\lambda \rightarrow \infty$.

We prove this fact at the end of this section.

Step 2. For $t>0, \Gamma f^{\lambda}(t) \rightarrow 0$ in $H_{\mathrm{loc}}^{N-1}(\bar{\Omega})$ as $\lambda \rightarrow \infty$.

In fact, this follows from Theorem A, Step 1 and

Lemma 3.1. Let $\left\{f_{n}\right\}_{n=1}^{\infty}$ be a bounded set in $H^{m}(\Omega)(m \geqq 1)$. Suppose that $f_{n} \rightarrow f$ weakly in $L^{2}(\Omega)$ as $n \rightarrow \infty$. Then $f_{n} \rightarrow f$ in $H_{\mathrm{loc}}^{m-1}(\bar{\Omega})$ as $n \rightarrow \infty$.

Proof. By Rellich's theorem, there exists a subsequence $\left\{f_{n^{\prime}}\right\}$ such that $f_{n^{\prime}}$ converges to some $g$ in $H_{\mathrm{loc}}^{m-1}(\bar{\Omega})$ as $n \rightarrow \infty$. But $f=g$, since $f_{n^{\prime}}$ converges weakly to $f$ in $L^{2}(\Omega)$. This shows that $f_{n}$ itself converges to $f$ in $H_{\mathrm{loc}}^{m-1}(\bar{\Omega})$.

Step 3. There exists a subsequence $\left\{\lambda_{v}\right\}$ such that $\Gamma_{0} f^{\lambda_{v}}(t)$ is convergent in $C\left(I ; H_{\text {loc }}^{N-1}(\bar{\Omega})\right)$. 
Indeed, by Theorem A, we have

$$
\sup _{\lambda>A, t \in I}\left(\left\|\Gamma_{0} f^{\lambda}(t)\right\|_{N}+\left\|\partial_{t} \Gamma_{0} f^{\lambda}(t)\right\|_{N-1}\right)<\infty .
$$

Then, by the Rellich and the Ascoli-Arzela theorems, there exists a subsequence $\left\{\lambda_{v}\right\}$ such that $\left\{\Gamma_{0} f^{\lambda_{v}}(t)\right\}$ is convergent in $C\left(I ; H_{\mathrm{loc}}^{N-2}(\bar{\Omega})\right)$. Recall the interpolation inequality: for a bounded set $B$ in $\Omega$, there exists a constant $C>0$ such that

$$
\|f\|_{H^{N-1}(B)} \leqq C\|f\|_{H^{N}(B)}^{1 / N}\|f\|_{L^{2}(B) / N}^{(N-1) / N} .
$$

This and Theorem A imply that

$$
\left\|\Gamma_{0} f^{\lambda}(t)-\Gamma_{0} f^{v}(t)\right\|_{H^{N-1}(B)} \leqq C\left\|\Gamma_{0} f^{\lambda}(t)-\Gamma_{0} f^{v}(t)\right\|_{L^{2}(B)}^{(N-1) / N},
$$

which shows that $\Gamma_{0} f^{v_{v}}(t)$ is convergent in $C\left(I ; H_{\mathrm{loc}}^{N-1}(\bar{\Omega})\right)$.

Step 4. Let $f^{\infty}(t)$ be the limit of $\Gamma_{0} f^{\lambda_{v}}(t)$. Note that $\sup _{t \in I}\left\|f^{\infty}(t)\right\|_{N-1}<\infty$. We show that

$$
f^{\infty}(t)=f_{0}^{\infty}-\int_{0}^{t} \Gamma_{0} A\left(f^{\infty}(s)\right) f^{\infty}(s) d s, \quad f_{0}^{\infty}={ }^{t}\left(0, P_{s} v_{0}^{\infty}\right) .
$$

In fact, by multiplying $\Gamma_{0}$ by (3.2) and taking the inner product with $g \in L^{2}(\Omega)$, we have

$$
\left(\Gamma_{0} f^{\lambda}, g\right)=\left(\Gamma_{0} f_{0}^{\lambda}, g\right)-\int_{0}^{t}\left(A\left(f^{\lambda}\right) f^{\lambda}, \Gamma_{0} g\right) d s .
$$

Then, we have only to approximate $\Gamma_{0} g$ by $h \in C_{0}^{\infty}(\Omega)$ and let $\lambda=\lambda_{\nu}$ tend to infinity.

Step 5. Step 4 implies that $f^{\infty}(t)={ }^{t}\left(0, v^{\infty}(t)\right), \quad v^{\infty}(t) \in C\left(I ; H_{\mathrm{loc}}^{N-1}(\bar{\Omega})\right)$, $\sup _{t \in I}\left\|v^{\infty}(t)\right\|_{N-1}<\infty$. Furthermore, $v^{\infty}(t)$ satisfies

$$
\begin{gathered}
\partial_{t} v^{\infty}(t)+P_{S}\left(v^{\infty}(t) \cdot \nabla\right) v^{\infty}(t)=0 \quad \text { in } \quad \Omega, \quad t \in I, \\
v^{\infty}(0)=v_{0}^{\infty}=P_{S} v_{0}^{\infty} .
\end{gathered}
$$

Now, we know that the above incompressible Euler equation has a unique solution, which shows that $f^{\lambda}(t)$ itself converges to ${ }^{t}\left(0, v^{\infty}(t)\right)$ in $H_{\mathrm{loc}}^{N-1}(\bar{\Omega})$ as $\lambda \rightarrow \infty$.

We turn to the proof of Step1. Taking the inner product of (3.2) with $g \in L^{2}(\Omega)$, we have

$$
\left(\Gamma f^{\lambda}(t), g\right)=\left(f_{0}^{\lambda}, e^{i t \lambda L} \Gamma g\right)-\int_{0}^{t}\left(A\left(f^{\lambda}\right) f^{\lambda}, e^{i(t-s) \lambda L} \Gamma g\right) d s .
$$

We show that for $t-s>0,\left(A\left(f^{\lambda}\right) f^{\lambda}, e^{i(t-s) \lambda L} \Gamma g\right) \rightarrow 0$ as $\lambda \rightarrow \infty$. Here we recall a notion in scattering theory. Let

$$
M=-i\left(\begin{array}{cc}
0 & \nabla \\
{ }^{t} \nabla & 0
\end{array}\right)
$$

be the linearized operator of acoustics in $L^{2}\left(R^{n}\right)$, and $\Gamma(M)$ the projection onto the orthogonal complement of the null space of $M$. Then by the completeness of wave operators (see, e.g., $[12,13]$ ), for any $f \in L^{2}(\Omega)$, there exist $f_{ \pm} \in L^{2}\left(R^{n}\right)$ such that

$$
\left\|e^{-i t L} \Gamma f-e^{-i t M} \Gamma(M) f_{ \pm}\right\|_{L^{2}(\Omega)} \rightarrow 0 \quad \text { as } \quad t \rightarrow \pm \infty .
$$


Therefore, we see that there exists an $h \in L^{2}\left(R^{n}\right)$ such that

$$
\left\|e^{i(t-s) \lambda L} \Gamma g-e^{i(t-s) \lambda M} \Gamma(M) h\right\|_{L^{2}(\Omega)} \rightarrow 0 \quad \text { as } \quad \lambda \rightarrow \infty,
$$

which implies that as $\lambda \rightarrow \infty$

$$
\left(A\left(f^{\lambda}\right) f^{\lambda}, e^{i(t-s) \lambda L} \Gamma g\right)=\left(\left(A\left(f^{\lambda}\right) f^{\lambda}, e^{i(t-s) \lambda M} \Gamma(M) h\right)+o(1) .\right.
$$

Now, for any $\varepsilon>0$, we choose an $h_{\varepsilon}$ such that $\hat{h}_{\varepsilon}=$ the Fourier transform of $h_{\varepsilon} \in C_{0}^{\infty}\left(R^{n}-\{0\}\right)$ and $\left\|h-h_{\varepsilon}\right\|<\varepsilon$. Then we have

$$
\left(A\left(f^{\lambda}\right) f^{\lambda}, e^{i(t-s) \lambda L} \Gamma g\right)=\left(A\left(f^{\lambda}\right) f^{\lambda}, e^{i(t-s) \lambda M} \Gamma(M) h_{\varepsilon}\right)+O(\varepsilon) .
$$

In view of Theorem $\mathrm{A}$, we have for a constant $C>0$,

$$
\left|\left(A\left(f^{\lambda}\right) f^{\lambda}, e^{i(t-s) \lambda L} \Gamma g\right)\right| \leqq C\left(\left\|e^{i(t-s) \lambda M} \Gamma(M) h_{\varepsilon}\right\|_{L^{\infty}(\Omega)}+\varepsilon\right)
$$

for large $\lambda$. The proof is thus completed if we show

$$
\left\|e^{i(t-s) \lambda M} \Gamma(M) h_{\varepsilon}\right\|_{L^{\infty}(\Omega)} \rightarrow 0 \quad \text { as } \quad \lambda \rightarrow \infty .
$$

The characteristic roots of $M$ are $\pm|\xi|$ and 0 . Let $Q_{ \pm}(\xi)$ and $Q_{0}(\xi)$ be the corresponding eigenprojections. Since the Fourier transform of $e^{-i t M} \Gamma(M) f$ is

$$
e^{-i t|\xi|} Q_{+}(\xi) \hat{f}(\xi)+e^{i t|\xi|} Q_{-}(\xi) \hat{f}(\xi)
$$

we have only to show the following lemma.

Lemma 3.2. Let $f(\xi) \in C_{0}^{\infty}\left(R^{n}-\{0\}\right)$. Then

$$
\left\|\int_{R^{n}} e^{i(x \xi-t|\xi|)} f(\xi) d \xi\right\|_{L^{\infty}\left(R^{n}\right)} \rightarrow 0 \quad \text { as } \quad|t| \rightarrow \infty .
$$

The proof of the above lemma follows from the method of stationary phase on the sphere, and is omitted.

In a similar manner, one can prove $\left(f_{0}^{\lambda}, e^{i t \lambda L} \Gamma g\right) \rightarrow 0$ as $\lambda \rightarrow \infty$ using the assumption (A-1). We have thus completed the proof of Theorem B.

\section{References}

1. Added, S., Added, H.: Asymptotic behavior for the solution of the compressible Navier-Stokes equation when the viscosity goes to 0 . Preprint 1986

2. Agemi, R.: The incompressible limit of compressible fluid motion in a bounded domain. Proc. Jpn. Acad. Ser. A 57, 292-293 (1981)

3. Asano, K.: On the incompressible limit of the compressible Euler equation. Preprint 1985

4. Da Veiga, Beirao: Stationary motions and incompressible limit for compressible viscous fluids. MRC Technical Summary Report No. 2883

5. Bourguignon, J.P., Brezis, H.: Remarks on the Euler equations. J. Funct. Anal. 15, 341-363 (1975)

6. Browning, G., Kreiss, H.O.: Problems with different time scales for nonlinear partial differential equations. SIAM J. Appl. Math. 42, 704-708 (1982)

7. Ebin, D.: Motions of slightly compressible fluids in a bounded domain. I. Commun. Pure Appl. Math. 35, 451-485 (1982)

8. Isozaki, H.: Singular limits for the compressible Euler equation in an exterior domain. J. Reine Angew. Math. (to appear) 
9. Kato, T.: Nonstationary flows of viscous and ideal fluid in $R^{3}$. J. Funct. Anal. 9, 296-305 (1972)

10. Klainerman, S., Majda, A.: Compressible and incompressible fluids. Commun. Pure Appl. Math. 34, 629-651 (1982)

11. Ladyzenskaya, O.A.: The mathematical theory of viscous incompressible flow. New York, London, Paris: Gordon and Breach 1969

12. Lax, P.D., Phillips, R.S.: Scattering theory. New York: Academic Press 1967

13. Mochizuki, K.: Spectral and scattering theory for symmetric hyperbolic systems in an exterior domain. Publ. RIMS. Kyoto University 1969, pp. 219-258

14. Majda, A.: Compressible fluid flow and systems of conservation laws in several space variables. Berlin, Heidelberg, New York: Springer 1984

15. Schochet, S.: The compressible Euler equations in a bounded domain: Existence of solutions and the incompressible limit. Commun. Math. Phys. 104, 49-75 (1986)

16. Schochet, S., Weinstein, M.: The non-linear Schrödinger limit of the Zakharov equations governing Langmuir turbulence. Commun. Math. Phys. 106, 569-580 (1986)

17. Ukai, S.: The incompressible limit and the initial layer of the compressible Euler equation. J. Math. Kyoto Univ. 26, 323-331 (1986)

18. Temam, R.: Navier-Stokes equations. Amsterdam, New York, Oxford: North-Holland 1977

Communicated by H. Araki

Received December 19, 1986 